\title{
Spanish norms for completion of restricted length word stems
}

\author{
María José Soler ${ }^{1,2} \cdot$ Carmen Dasí $^{1}$ • Juan Carlos Ruiz ${ }^{1}$. Teresa Cervera ${ }^{1}$
}

Published online: 6 December 2016

(C) Psychonomic Society, Inc. 2016

\begin{abstract}
This study presents a normative database of Spanish restricted length word stems that provides useful information for the selection of stimuli in memory experiments with Word Stem Completion (WSC) tasks. The database includes indices relative to stems (total baseline completion, priming baseline completion, priming, number of completions, ratio between given and deleted letters, and syllabic structure), and indices relative to characteristics of the words used to obtain the stems (frequency, familiarity, number of meanings, length, number of syllables, arousal, and valence). A WSC task was performed by 515 participants to calculate priming and baseline indices. An Exploratory Factor Analysis showed that these indices are grouped in four factors: perceptual, lexical, emotional, and response competition. Stepwise regression analyses performed with these factors showed that the lexical, response competition, and perceptual factors predict priming baseline completion, while only the lexical factor predicts priming. The model that best explains the relationship between priming and priming baseline completion was a cubic model, and the optimum baseline values for achieving priming were between .31 and .36 . These norms can be downloaded as Supplemental Materials for this article from https://nuvol.uv.es/owncloud/index.php/s/hpj9bylqbENdjfj.
\end{abstract}

Electronic supplementary material The online version of this article (doi:10.3758/s13428-016-0834-x) contains supplementary material, which is available to authorized users.

María José Soler

Maria.J.Soler@uv.es

1 Faculty of Psychology, University of Valencia, Valencia, Spain

2 Facultad de Psicología, Avda. Blasco Ibañez, 21, 46010 Valencia, Spain
Keywords Word stems · Normative database $\cdot$ Implicit memory $\cdot$ Priming baseline completion $\cdot$ Priming

Word completion tasks are widely used to study different aspects of implicit memory in clinical and non-clinical populations (Geraci \& Hamilton, 2009; Marques, Spataro, Cestari, Sciarretta, \& Rossi-Arnaud, 2016; Mitchell \& Bruss, 2003; Millet, Le Goff, Bouisson, Dartigues, \& Amieva, 2010; Pomarol-Clotet, Oh, Laws, \& McKenna, 2008). In these studies implicit memory has been evaluated through repetition priming, usually defined as the benefit in the processing of a stimulus when it has been recently processed (Roediger \& Blaxton, 1987). When a word completion task is used to study priming, a two-phase structure is usually followed. In an initial phase, called the study phase, a list of words (called "primes") are presented to the participants to be processed without a conscious study of them (they are not instructed to study the primes); for example, subjects are asked to judge the familiarity of the words. In a second phase, called the test phase, participants view an incomplete word (called the "target") and are asked to complete it with the first word that comes into their mind. The nature of the test phase is unspecified in the first phase. In the second phase, some of the targets are words processed in the first step of the task, though the instructions do not make any explicit reference to this, and other targets are words not seen in the study phase. Priming is defined as the increase in the proportion of completions of words previously presented over the proportion of completions of words not presented in the study phase.

The most common targets used in word completion tasks are three-letter word beginnings (Brooks \& Gibson, 2012). According to Gibson and Watkins (1988), one reason why these targets are so widely used is the relative ease with which one can confirm - using a dictionary - how many potential 
responses correspond to each stem. The availability of information about the number of possible completions of a stem is very convenient when these stimuli are selected for use in research about implicit memory, because this variable has an effect on the probability of completing the stem (Gibson, 2012), and also because said probability of stem completion is used to calculate the priming. This information is much more difficult to achieve when other types of targets are used, such as the word fragments used in Word Fragment Completion (WFC) tasks; unlike stems constituting the beginning of a word, in the case of fragments some letters of a word are randomly removed and are replaced by underscores.

Word-beginning stems are characterized by the fact that they can be pronounced and usually provide a syllabic unit that helps their completion, as the lexicon is organized in alphabetical order, like a dictionary. Word-beginning stems can be of unconstrained or constrained-length (Mueller \& Thanasuan, 2014). In the case of unconstrained-length stems, participants are asked to complete the stem with any word, independently of its length (e.g., correct completions to the stem "mel" are "melt" or "melodrama"). However, in constrained or restricted-length stems the remaining letters of the word are replaced by underscores (e.g,. a correct completion of the stem "sol _ _ _ " is "soldier", but not "solarium"); that is, a completion is correct only when the response has the same beginning as the stem and when it fulfils an indicated length.

Unconstrained-length stems are the most used stems in Word Stem Completion (WSC) tasks. One of the results observed with these stems is that they are solved more easily and faster than other targets used in implicit tasks, such as fragments. According to Roediger, Weldon, Stadler, and Riegler (1992), this result occurs because stems usually have multiple completions - more than ten in many studies - and this favors the completions being accomplished with highly frequent and short words, thus making the tasks relatively easy. In fact, in the English language, few experiments use unconstrainedlength stems with only one completion (Barnhardt, 2005). However, the use of unconstrained-length stems may have two disadvantages: (a) a high variability and heterogeneity of answers from participants, since there is usually a large number of correct alternatives; and (b) an underestimation of priming, as priming seems to depend on the amount of possible completions of the stems (Barnhardt, 2004, 2005).

A high variability in responses was observed in the study of Martin, Dressaire, Guerdoux, Trouillet, Brouillet, Brouillet, and Maury (2009), which used unconstrained stems of French words. In 3,276 different participant answers, only one response achieved a degree of agreement of $60 \%$, and only 18 responses obtained a degree of agreement above 50 $\%$. Martin et al. (2009) proposed that one way to avoid the high variability of responses and, thus, maximize priming, is to use constrained or restricted-length stems, which means limiting completions to a particular word length. According to Martin et al. (2009), limiting word length avoids an overestimation of participant performance. If there is no length limitation, participants can use strategies that lead them to generate very long or infrequent words that were not processed previously in the task. However, if constrained-length stems are used, it is easier to control the number of alternative responses, and also to identify a priori those alternatives and their linguistic characteristics (e.g., frequency, length, etc.). For this reason, in their normative study, Martin et al. (2009) replaced unconstrained stems with constrained stems for which the only responses were five-letter words.

Moreover, a high variability in response alternatives may have an undesired effect, the underestimating of the influence of experimental variables on priming; especially when the changes in the magnitude of priming are relatively small and difficult to observe. Therefore, minimizing the variability of responses to stems may be a strategy to maximize the conditions in which priming occurs and to help selecting stimuli with the potential capacity to generate priming. This may be particularly relevant in the study of implicit memory in some clinical populations. In recent decades numerous studies using word completion tasks in clinical subjects, such as Alzheimer disease or schizophrenic patients (e.g., Fleischman, 2007; Soler, Ruiz, Vargas, Dasí, \& Fuentes, 2011), have reported contradictory priming results, and it has been hypothesized that the characteristics of the stimuli used in the tasks could have been responsible for the heterogeneous results obtained. For example, it has been observed that Alzheimer patients show unimpaired performance in completion tasks under highly restricted situations in which there is only one alternative response; however, the same patients performed poorly under unrestricted situations, or when there were many possible answers. The unimpaired ability of these subjects at certain stages of the disease has been associated with different types of tasks, such as category labels or incomplete sentences and in word stem completion tasks (Hartman \& Pirnot, 1995; Nebes, 1989). Studies with schizophrenic patients (e.g., Marvel, Schwartz, \& Isaacs, 2004) have replicated these results, showing selective deficits in word stem completion in the condition of few completions. Therefore, the availability of constrained stems can help with the assessment of implicit memory deficits, and to identify priming differences between patients and healthy populations.

At the same time, studies analyzing the effect of normal and abnormal aging on implicit memory can also benefit from using constrained stems. Research has produced contradictory results when different types of tasks have been used to assess priming (Ryan, Ostergaard, Norton, \& Johnson, 2001). The study by Martin et al. (2009) observed an effect of age on the variability of responses in completing unconstrained stems, which could help to understand contradictory results. In their study, carried out in five different age groups ranging from 
$30-40$ to $\geq 70$ years, the variability in the number of responses to unconstrained stems increased as participant age rose. This finding underlines the need to use constrained stems in WSC tasks to control variability of responses.

The above-mentioned results show that stimuli involved in completion tasks must be carefully selected to control potentially confounding variables that can lead to the underestimation of the effects of priming (Gibson, 2012). However, many studies do not provide enough information about how authors choose the original words for generating the experimental stimuli, or only make a general reference to the frequency of the words, or the mean number of completions of the stems. This lack of information suggests that these studies may not have controlled relevant variables related with characteristics of the stimuli and that their results may have been misinterpreted.

Evidence of the effects of psycholinguistic characteristics of words and stems - such as frequency, familiarity or length, and number of completions - on the probability of stem completion and priming has been obtained with constrained and unconstrained stems. In the case of word frequency and familiarity, some studies have found a positive correlation between these variables and the probability of constrained stem completion (e.g., Martin et al., 2009); that is, stems obtained from high-frequency words are completed more easily than those obtained from low-frequency words. At the same time, the relation between frequency and completion probability has been reported in some studies using unconstrained stems (Graf \& Williams, 1987; Mueller \& Thanasuan, 2014), although others have failed to find such a relation (Migo, Roper, Montaldi, \& Mayes, 2010). Word length is another variable that seems to have some influence on the probability of completion of unconstrained stems (Graf \& Williams, 1987), and this effect has been replicated with constrained stems, even though "the true role of length in stem completion remains somewhat difficult to determine" (Mueller \& Thanasuan, 2014). The relationship between the number of completions of stems and their completion probability has not been systematically studied. Only a few studies have been conducted with unconstrained stems, some of which reported that the probability of completing a stem was higher in unique versus multiple completions (Gibson, 2012; Marques et al., 2016).

The effects of these variables on priming scores have only been studied with unconstrained stems, and results indicate that the frequency and familiarity of the words correlate with priming scores; i.e., high-frequency words and highly familiar words produce lower priming scores than low-frequency words and unfamiliar words (Roediger et al., 1992). Studies on the effect of number of stem completions on priming have produced contradictory results. Some have reported that priming decreases as the number of possible stem completions increases (Barnhardt, 2004; Nyberg, Winocur, \&
Moscovitch, 1997); others have found that the decrease is quite small (Gibson, 2012), while some have not found differences when comparing few and many possible completions stems (Geraci \& Hamilton, 2009; Jacoby, Toth, \& Yonelinas, 1993; Marques et al., 2016). Besides the effects of the psycholinguistic characteristics of words on priming reported in the literature, some studies have shown that there are emotional dimensions of words that also have an effect on priming. In unconstrained word stem completion tasks, a dissociative effect of the affective dimension of words on the priming has been observed when healthy controls are compared with patients with depression (Fernández-Rey \& Merino, 2002), patients with anxiety disorder (Mathews, Mogg, May, \& Eysenck, 1989) and with Alzheimer patients (Keane et al., 1991). Literature shows that emotional arousal also decreases priming in unconstrained stem completion tasks (Kellerman, 2002).

In summary, it seems necessary to have word stem normative bases, first, to facilitate the selection of stimuli for research purposes, and, second, to advance in clarifying how the characteristics of these stimuli help to explain the probability of stem completion and priming. A review of the literature concerning word stem completion norms reveals that there are norms of unconstrained stems of English (Graf \& Williams, 1987; Migo, et al., 2010) and Swedish (Olofsson \& Nyberg, 1992) words, and of constrained or restricted stems of five-letter French words (Martin et al., 2009) and five- to seven-letter German words (Bergmann, Müller, \& Danek, 2010). In Spanish, the only available database is that developed by Soler, Dasí, and Ruiz (2009), which has 269 unique solution word fragments. This database provides useful information for the selection of stimuli in implicit memory experiments with WFC tasks, such as the indices of priming and completion probability for each fragment. However, there is no currently published normative database of Spanish word stems. Therefore, the main objective of this study is to provide researchers with a normative database of Spanish restricted length word stems. The absence of norms for completion of Spanish word stems hinders the appropriate selection of this type of stimuli for research. We consider that the information included in the database - indices such as priming and completion probability of stems - will help Spanish-speaking researchers in the study of implicit memory and will allow them to control potentially confounding variables and explore experimental effects (e.g., aging, divided attention) (Geraci \& Hamilton, 2009; Spataro, Cestari, \& Rossi-Arnaud, 2011; Spataro, Mulligan, \& Rossi-Arnaud, 2010). Finally, the information provided by the database may facilitate the establishment of a standard implicit memory test to be used in the future by applied psychologists.

The normative base of constrained stems of Spanish words presented in this study uses the Soler et al. (2009) database as a starting point. The same words used to elaborate the 
fragments included in said database have been employed to obtain constrained stems. This procedure has allowed us to obtain information with which to make comparisons between the two types of stimuli (stems and fragments) and tasks (WSC and WFC). For example, if we know the number of possible completions the stem and the fragment obtained from a specific word, we can determine the stimuli that have the same number of possible completions in both cases. The lack of such information about these stimuli has been mentioned as a limitation in some studies (e.g., Fay, Isingrini, \& Clarys, 2005).

To achieve our main objective, a constrained WSC task was first administered to a sample of participants to collect the necessary data to calculate, for example, the indices of priming and completion probability for each stem. Secondly, the database was completed with additional information relative to the stems (e.g., number of completions or syllabic structure), psycholinguistic characteristics of the words that were used to obtain the stems (e.g., frequency or length), and emotional dimensions of these words (e.g., affective valence). Our research goes further by performing additional analyses of this information to try to identify which characteristics of the words and stems are the best predictors of priming and completion probability. Finally, we compare the indices obtained in the WSC task with those obtained in a previous WFC study (Soler et al., 2009).

\section{Method}

\section{Participants}

Five hundred and fifteen undergraduate native Spanishspeaking students enrolled in the University of Valencia's introductory psychology courses took part in the study. All participants had normal or corrected-to-normal vision, and their age ranged between 18 and 45 years $(\mathrm{M}=22,25 ; \mathrm{SD}=6,70)$.

\section{Materials}

A constrained word stem task was used. The stimuli in the task were the words included in the Soler et al. (2009) database. This normative base contains a total of 269 words with corresponding unique solution fragments and includes several indices associated with each of the words, and several indices associated with each of the fragments. These words have between five and seven letters because Spanish words of fewer than five letters are not appropriate to obtain three-letter stems or fragments, as a ceiling effect will appear in WSC and WFC tasks. Moreover, words of more than seven letters are not very common in Spanish.

Each of the stems included the three first letters of the word, and the remaining letters were replaced by underscores. Unlike what happened with the fragments, the word stems had one or more possible completions. Because the words were the same as those used by Soler et al. (2009) to make unique fragments, some of the three-letter word stems obtained were identical for more than one word. Therefore, the total number of intended words was 269 , and the number of different stems obtained from these words was 255 .

To find the possible completions for each stem, a search was conducted using the Dictionary of the Royal Spanish Academy of Language (2001). A completion was valid if it morphologically matched a common Spanish name (in singular) and had a length (number of letters) equal to that of the word that was used to generate the stem (e.g., from the Spanish word "caballo" the stem was "c a b___,, and this stem has nine more valid completions: "caballa, cabeceo, cabello, cabezal, cabezón, cabildo, cabrera, cabrero, cabrito"; the fragment in Soler et al. (2009) for the word "caballo" was "c__a_ 1o"). Thus, plurals, verbs, and verbal forms were not valid completions; however, both genders of common names were considered valid completions. Only words with frequency values greater than 0 were selected.

\section{Procedure}

A WSC task was administered to obtain the baseline completion and priming indices for the stems. The procedure followed by Soler et al. (2009) was replicated in order to compare the two indices obtained in the WFC task and in the WSC task. This procedure consisted of an initial study phase in which a list of words in lowercase was presented in the center of a screen, one by one, for a total of $8 \mathrm{~s}$ each. Participants had to judge the familiarity of each of these words (primes) on a scale ranging from 1 (unfamiliar) to 7 (very familiar), according to the procedure of Erickson, Gaffney, and Heath (1987). Afterwards, in the test phase, a list of word stems in lowercase was presented, in the center of the screen, one by one, for $12 \mathrm{~s}$ each. Half of the stems belonged to words that had been evaluated in the first phase of the task and half belonged to new words. Participants were requested to write on an answer sheet the first word that came into their minds upon seeing each stem, and this word had to be a singular common word in Spanish language. In this phase there was no explicit reference to the initial evaluation of word familiarity. Between the first and second phases participants performed a 5-min distractor task in which they had to write down the name of European cities. The WSC task was conducted in small groups of 25-35 students, and no participant had to complete more than 90 stem words; thus, the number of participants that evaluated each stimulus varied, averaging 86.15 ( $\mathrm{SD}=6.02$; range: 82-92). The same stimuli appeared for half of the participants as studied and for the other half as non-studied. 


\section{Indices included in the normative database}

The normative database, available online in the Supplementary Material, includes the following indices for stems and words.

\section{Indices for stems}

Total baseline completion The total proportion of completions with any word, in the condition in which the word has not been presented in the first phase of the task. It is the stem completion probability, and can be considered a measure of the difficulty of completion of a stem.

Priming baseline completion The proportion (or probability) of completions with the intended word (the word from which the stem was obtained) in the condition in which the word has not been presented in the first phase of the task.

Priming The difference between "priming baseline completion" and the completion probability of the stem with the intended word, when the word has been presented in the first phase of the WSC task.

Number of completions The total number of different words or valid completions of a stem that appear in the Dictionary of the Royal Spanish Academy of Language (2001).

Letters-blanks ratio Refers to the quotient between the letters in the stem and the deleted letters.

Syllabic structure This indicates if the stem corresponds with the first syllable of the intended word (one-syllable stem) or not (no-syllable stem), according to the Royal Spanish Academy of Language (2011).

\section{Indices for words}

Frequency The relative frequency in the Guasch, Boada, Ferré, and Sánchez-Casas (2013) lexical database, which has a total body of 5,629,279 Spanish tokens. When a word has a frequency of 0 it means that it is a word not included in that body.

Familiarity Defined as the everyday word occurrence frequency in spoken or written form, estimated by a sample of subjects and measured on a 7-point scale. This index was obtained from the Soler et al. (2009) database.

Number of meanings Indicates the number of meanings of each word corresponding to a common noun in singular. This information was obtained from the Dictionary of the Royal Spanish Academy of Language (2001). Some technical words did not appear in this dictionary, in which case the number of meanings was counted as 0 , but these words can be found in technical-scientific dictionaries such as the Dictionary of the Royal Academy of Sciences (1999).

Length Total number of letters in a word.

Number of syllables Number of syllables in a word.

Arousal Defined as the degree of activation from calming to exciting produced by a particular encounter with a word. This index was obtained from the Soler et al. (2009), and Redondo, Fraga, Comesaña, and Perea (2005) databases. This index is measured using a 9-point Likert scale $(1=$ calm; $9=$ excitement).

Valence Defined as the pleasantness associated with the experience of a word (Lang, 1980; Osgood, Suci, \& Tannenbaum, 1957). This index was obtained from the Soler et al. (2009), and Redondo et al. (2005) databases. This index is measured using a 9-point Likert scale ( 1 = unpleasant; $9=$ pleasant).

\section{Results and discussion}

The main descriptive statistics (range, mean, standard deviation, and standard error) of the different indices for the words and stems are shown in Table 1 . Seven words presented random (near 0) negative priming ("familia":-.04; "consejo": -.03; "noche": -.03; "refugio": -.02; "ilusión": -.0004; "vaguada": -.0004; "pensión": -.0002) and were converted to 0 , following the Soler et al. (2009) procedure for fragments.

The P-P and Q-Q graphical analyses for testing normality showed the presence of two variables with strong positive asymmetry: Frequency and number of completions. These variables were logarithmically transformed $\log (1+\mathrm{x})$, following the method from Cuetos and Alija (2003), and Dasí, Soler, and Ruiz (2004). The transformed data were used in the statistical analysis.

To verify the effect of priming in our stimuli a one-sample $t$-test was performed to ascertain if the effect was significant. The $t$ test (using the contrast value 0) showed that priming was significant $(t(268)=26.64, \mathrm{p}<.001 ; \mathrm{d}=1.62)$. This magnitude of priming $(\mathrm{M}=.22, \mathrm{SD}=.13)$ is in line with the results obtained in some previous studies with stems and with fragments (Roediger et al., 1992; Soler et al., 2009; Srinivas \& Roediger, 1990). 
Table 1 Descriptive statistics of indices for words and stems

\begin{tabular}{|c|c|c|c|c|c|}
\hline & Minimum & Maximum & Mean & SD & SE \\
\hline \multicolumn{6}{|l|}{ Indices for words } \\
\hline Frequency & .00 & 672.02 & 33.45 & 63.27 & 3.86 \\
\hline Familiarity & 1.02 & 6.75 & 4.60 & 1.67 & .10 \\
\hline Number of meanings & .00 & 27.00 & 4.50 & 3.94 & .24 \\
\hline Length & 5.00 & 7.00 & 6.19 & .75 & .05 \\
\hline Number of syllables & 2.00 & 4.00 & 2.72 & .56 & .03 \\
\hline Arousal & 2.14 & 8.20 & 4.99 & 1.06 & .06 \\
\hline Valence & 1.64 & 8.45 & 5.29 & 1.36 & .08 \\
\hline \multicolumn{6}{|l|}{ Indices for stems } \\
\hline Total baseline completion & .00 & 1.00 & .55 & .27 & .02 \\
\hline Priming baseline completion & .00 & .99 & .33 & .30 & .02 \\
\hline Priming & .00 & .60 & .22 & .13 & .01 \\
\hline Number of completions & 1.00 & 31.00 & 5.35 & 5.86 & .36 \\
\hline Letters-blanks ratio & .75 & 1.5 & 1.00 & .27 & .02 \\
\hline
\end{tabular}

$S D$ standard deviation, $S E$ standard error

\section{Correlation between indices}

The second step of our analyses was carried out to identify the relation between indices related with the following characteristics:

a. Words: Frequency, familiarity, number of meanings, length, number of syllables, arousal, and valence.

b. Stems: Priming baseline completion, priming, number of completions, and letters-blanks ratio.

The total baseline completion score was not considered in the analysis of correlation with the other variables. This index, as stated above, is obtained from the total proportion of completions of a stem, and provides information on its level of difficulty. This information is of real relevance when a researcher needs to perform a controlled selection of stimuli, however, for multiple completion stems it would be incorrect to calculate the correlation between total baseline completion and variables such as frequency, familiarity, or length, because these characteristics correspond with only one of the possible completions of the stem. For this reason the variable used in all the following analyses was priming baseline completion.

The Pearson correlation matrix appears in Table 2 and shows some significant associations between variables. First, expected significant positive correlations between variables were observed when we measured similar lexical characteristics of word representations in the memory, such as their frequency and familiarity $(r=.76)$, or frequency and number of meanings $(r=.55)$. Second, there was another group of significant correlations between variables with respect to more perceptive information of words and stems. For instance, there was a positive correlation between length and number of syllables $(r=.58)$, and a negative one between word length and letters-blanks ratio $(r=-.98)$. Third, a significant correlation was observed between the two indices assessing the affective dimensions of the words; e.g., valence and arousal $(r=-.19)$.

With respect to the priming baseline completion variable, the correlation matrix revealed several interesting associations. First, significant positive correlations were found with frequency $(r=.49)$, familiarity $(r=.53)$ and number of meanings $(r=.23)$, confirming that the stems obtained from words about which there is more information available in the memory were also easier to complete with the expected word (Mueller \& Thanasuan, 2014). Likewise, there was a positive correlation between baseline and valence of words $(r=.13)$; there was a higher probability of completing the stems of words that represented more pleasant experiences. Second, as expected, the results showed that the probability of completing a stem was negatively related with the number of letters $(r=-.25)$, and number of syllables in the stimuli $(r=$ -.13) (Graf \& Williams, 1987). Third, there was a negative correlation between baseline and number of completions of the stem $(r=-.46)$. This result suggests that when the number of possible alternatives for completion of a stem is high, the probability of choosing the intended word is lower.

The correlation matrix also showed the following significant associations between priming and the other variables. First, priming was negatively correlated with the number of completions of the stem $(r=-.16)$, indicating that targets having only one or few potential completions are those that produce more priming. This result is interesting, as it suggests that the number of possible completions of the stems should be considered when the priming is the dependent variable in an investigation (Soler, Dasí, \& Ruiz, 2015). Second, priming 
Table 2 Pearson linear correlations between indices

\begin{tabular}{|c|c|c|c|c|c|c|c|c|c|c|}
\hline & 1 & 2 & 3 & 4 & 5 & 6 & 7 & 8 & 9 & 10 \\
\hline 1. Frequency & - & & & & & & & & & \\
\hline 2. Familiarity & $.76^{* *}$ & - & & & & & & & & \\
\hline 3. Number of meanings & $.55^{* *}$ & $.46^{* *}$ & - & & & & & & & \\
\hline 4. Length & -.03 & -.05 & -.04 & - & & & & & & \\
\hline 5. Number of syllables & $-.21 * *$ & $-.15 *$ & $-.25 * *$ & $.58 * *$ & - & & & & & \\
\hline 6. Arousal & $.14 *$ & $.21 *$ & .06 & .00 & -.00 & - & & & & \\
\hline 7. Valence & $.31 * *$ & $.34 * *$ & $.18 * *$ & .01 & .05 & $-.19 * *$ & - & & & \\
\hline 8. Priming baseline completion & $.49 * *$ & $.53 * *$ & $.23 * *$ & $-.25^{*}$ & $-.13 *$ & .09 & $.13 *$ & - & & \\
\hline 9. Priming & $-.18 * *$ & -.12 & -.09 & -.10 & -.03 & -.02 & -.12 & $-.21 *$ & - & \\
\hline 10. Number of completions & .02 & .08 & .09 & $.30^{*}$ & .03 & .00 & .04 & $-.46^{* *}$ & $-.16 * *$ & - \\
\hline 11. Letters-blanks ratio & .04 & .06 & .04 & $-.98 * *$ & $-.57 * *$ & .01 & -.01 & $.27 * *$ & .07 & $-.31 * *$ \\
\hline
\end{tabular}

$* p<.05 ; * * p<.01$

correlated negatively with word frequency $(r=-.18)$, which is in line with previous results indicating that the highest magnitude of priming corresponds with words with the lowest frequency (Roediger et al., 1992). Third, there was a negative correlation between priming and valence $(r=-.12)$. The reason for this could be that the impact of the studying of a prime is greater in the case of words associated with less pleasant experiences.

Due to the importance of the relation between priming baseline completion and priming, further analyses were performed and are presented in the next section.

\section{Priming and baseline completion Indices}

An Exploratory Factor Analysis (EFA), using the method of principal components, was performed to analyze in depth if the variables included in the correlation matrix could explain priming and priming baseline completion. EFA was carried out for the remaining nine indices. The Kaiser-Meyer-Olkin (KMO) index for sample adequacy was .65; therefore, it was considered correct to perform an EFA for these data. As usual, a Varimax rotation with the Kaiser criterion of eigenvalues above 1 was used. The first analysis revealed a factor with an eigenvalue of .975 , which explained more than $10 \%$ of the variance, and so it was decided to include this factor (Table 3).

The grouping of variables highlighted the following factors. First, a factor called perceptual related to the amount of information provided by the word or stem. This factor groups the variables word length, number of syllables, and lettersblanks ratio of the stem. Second, a lexical factor based on the variables such as frequency of use, familiarity and number of meanings. Third, an emotional factor including the arousal and valence variables that make reference to the processing of affective aspects associated with the words. The fourth factor, called response competition factor, refers solely to the number of completions of the stems. All components accounted for $79.32 \%$ of the variance, with the following distribution: 29.94 $\%$ was perceptual component, $25.45 \%$ was lexical component, $13.09 \%$ was emotional component, and $10.84 \%$ was competition response component.

These four latent variables were included in a Stepwise Multiple Regression analysis carried out to explore their capacity to predict priming baseline completion. The contribution of three of them reached significance. First, the lexical component $(\beta=.48, p<.001)$ accounted for $22.80 \%$ of baseline variance. So, the most important factor explaining the probability of completing a stem with the intended word was the amount of lexical information about this word available in the memory. This information will have been accumulated through all prior encounters with the word, independently of the experimental situation (Ostergaard, 1998). Words with lexical representations that are more complete in memory are also those for which it is easier to find the intended completion of their stem. Second, the competition response component $(\beta=-.47, p<.001)$ accounted for $22.00 \%$ of the baseline variance. The influence that the number of

Table 3 Results of the rotated factorial solution of the Exploratory Factor Analysis

\begin{tabular}{lllll}
\hline & Factor 1 & Factor 2 & Factor 3 & Factor 4 \\
\hline Length & .93 & .00 & .02 & .25 \\
Number of syllables & .81 & -.19 & -.04 & -.25 \\
Letter-blank ratio & -.92 & .01 & -01 & -.26 \\
Familiarity & -.02 & .89 & .04 & -.03 \\
Frequency & -.04 & .90 & .01 & -.03 \\
Number of meanings & -.14 & .71 & -.01 & .23 \\
Valence & .11 & .47 & -.67 & -.17 \\
Arousal & .06 & .24 & .86 & -.13 \\
Number of completions & .18 & .08 & -.02 & .90 \\
\hline
\end{tabular}


completions of the stem has on its probability to be completed with the intended word is relevant. This result indicates the need to take into account the variable number of completions in the selection of stimuli for studies on priming, especially in implicit memory experiments. Third, the perceptual component $(\beta=-.17, p<.001)$ accounted for $3.00 \%$ of the baseline variance, suggesting that the amount of information provided to solve the target (e.g., letters-blanks ratio), or physical characteristics of the words (e.g., length) explain the probability of completing the stems to a lesser extent than the previous two factors.

A further Stepwise Multiple Regression Analysis was performed with the same four components, but considering priming as a dependent variable. In this analysis only one component was significant; namely, the lexical component, which explained $2.70 \%$ of the variance $(\beta=-.16, p<.01)$. The unique variable included in this lexical factor, which showed a significant correlation with priming, was word frequency ( $r$ $\left.=-.18, p<.01 ; \mathrm{R}^{2}=0.03\right)$. Its capacity to explain priming in WSC tasks, as we have now observed, has been also shown in other experimental studies (Rajaram \& Roediger, 1993; MacLeod, 1989; MacLeod \& Kampe, 1996; Roediger et al., 1992). However, according to Nelson and McEvoy (2000) there is a debate about the interpretation of what measures "word frequency." These authors elaborated free association norms of words and they analyzed the relations between frequency and other psycholinguistic word characteristics. Results showed a relationship between frequency and "accessibility" defined as the "number of times that each normed word was produced as a response by another normed word". Then, it could be hypothesized that frequency seems to reflect not only a perceptual word processing, but also that it is an index related to the accessibility to the lexical representation of the word in memory.

The factor that included the variable number of completions was not significant, however this variable showed a significant correlation with priming $\left(r=-.16, p=.01 ; \mathrm{R}^{2}=0.03\right)$. The role of this variable in predicting priming is currently under debate, as its influence has been observed in some studies (e.g., Soler et al., 2015), but not in others (e.g., Gibson, 2012). All these results suggest once again that the variable number of completions should be a criterion when selecting stimuli in WSC tasks.

As stated above, we have analyzed with special attention the relationship between priming and priming baseline completion. The model that best fits the relationship between these variables is the cubic equation, which explains $30 \%$ of the variance in priming $(F(3,265)=38.04, p<.001)$. The regression equation is $\mathrm{y}^{\prime}=.16+.95 \mathrm{x}-1.75 \mathrm{x}^{2}+.63 \mathrm{x}^{3}$. The inflection point corresponds with a baseline of .33 , with optimum baseline values ranging between .31 and .36 , with a priming of around .31 , and a sharp decline in priming when the baseline exceeds 0.65 ; e.g., with easy stems. Therefore, in experiments with WSC tasks, it is recommended to use stems with high or medium competition probability in order to maximize priming.

\section{Comparison of results from WSC and WFC tasks}

The last objective of our study was to compare the priming baseline completion and the priming obtained using WSC and WFC tasks. As previously described, in order to make a direct comparison between the two tasks, we replicated the procedure followed by Soler et al. (2009) in a WFC task, and the stems were obtained from the same words as the fragments. Thus, we now have information on priming baseline completion and priming for the 269 words, both in their form as a stem or as a fragment. In terms of priming, we have observed that both tasks have the capacity to generate this effect with a similar magnitude. The average for priming, considering all the stimuli in the database, is .20 for fragments, and .22 for the stems. In the case of priming baseline completion, the average for the fragments is .42 and .33 for the stems. However, this comparison must take into account that one of the variables influencing baseline is the number of completions of the intended word, and that, while fragments always have a unique solution, stems may have more than one completion. If we consider only the stems of the database with a unique completion, and we compare the baseline completion of these stems with that of the corresponding fragments, the results show averages to be almost the same ( $\mathrm{M}$ for fragments $=.49$; and $\mathrm{M}$ for stems $=.51)$.

Furthermore, the relationship observed between the two variables (priming and priming baseline completion) proved to have an identical profile in the two tasks. As stated regarding fragments by Soler et al. (2009), the model that best fits this relationship in the case of stems is also the cubic equation. Fragments and stems that produce more priming are those with a baseline completion around .30. In other words, the processing of stimuli with a moderate to high level of completion probability has a greater impact on its further processing; similarly, in both tasks, easy stimuli are those that have less capacity to generate priming.

The factorial structure of the two tasks was almost identical, with the exception of the number of completions, which is a variable associated only with stems because all the fragments had a unique solution. The factorial structure showed that the lexical and perceptual factors predicted baseline completion for priming in both tasks; that is, the factors that explain the probability of completing fragments and stems are the same, and are related to: (a) the availability of the information of a word that we have in the memory; and (b) the recovery keys 
provided for completing the target. Regarding the regression analysis using the factors as predictors of priming in both WSC and WFC tasks, only the lexical component was statistically significant.

\section{Conclusions}

Present norms provide information that will help Spanishspeaking researchers in the study of memory and other cognitive domains. There are only available norms of unconstrained stem completion of English words (Graf \& Williams, 1987; Migo, et al., 2010) and Swedish (Olofsson \& Nyberg, 1992), and of constrained stems of French words (Martin et al., 2009) and German words (Bergmann et al., 2010). However, at the present time, there are no norms for completion of constrained and unconstrained Spanish word stems. We believe it is especially interesting that these norms include indices such as the number of possible completions of the stems, the probability to complete a stem with any of the possible responses (total baseline completion), the probability of completion a stem with an intended response (priming baseline completion), or the priming. This information will help researchers to make an appropriate selection of stimuli to control of potentially confounding variables and to study experimental effects. The availability of those indices for stems and fragments, from the same words, allows the comparison of results from WFC and WSC tasks. Finally, the information provided by these norms may facilitate the development of a standard implicit memory test to be used in the future by applied psychologists.

\section{References}

Barnhardt, T. M. (2004). Different involuntary mechanisms underlie priming and LOP effects in stem completion tests. Memory, 12, 614-636. doi:10.1080/09658210344000143

Barnhardt, T. M. (2005). Number of solutions effects in stem decision: Support for the distinction between identification and production processes in priming. Memory, 13, 725-745. doi:10.1080/09658210444000368

Bergmann, H. C., Müller, B. C. N., \& Danek, A. (2010). Normdaten zur Ergänzung deutscher Wortanfänge (Normative data for the completion of German word stems). Zeitschrift für Neuropsychologie, 21, 17-23. doi:10.1024/1016-264X/a000003

Brooks, J. O., III, \& Gibson, J. M. (2012). Implicit memory is not a unitary construct: factor analysis of eight implicit memory measures. In N. Hsu \& Z. Schütt (Eds.), Psychology of priming (pp. 245-263). New York: Nova Science Publishers, Inc.

Cuetos, F., \& Alija, M. (2003). Normative data and naming times for action pictures. Behavior Research Methods, Instruments, \& Computers, 35, 168-177. doi:10.3758/BF03195508

Dasí, C., Soler, M. J., \& Ruiz, J. C. (2004). Normative data on the familiarity and difficulty of 196 Spanish word fragments. Behavior Research Methods, Instruments, \& Computers, 36, 559-563. doi:10.3758/BF03195602
Erickson, J. R., Gaffney, C. R., \& Heath, W. P. (1987). Difficulty and familiarity norms for 192 single-solutions word fragments. Behavior Research Methods, Instruments, \& Computers, 19, 370-376. doi:10.3758/BF03202580

Fay, S., Isingrini, M., \& Clarys, D. (2005). Effects of depth-of-processing and ageing on word- stem and word-fragment implicit memory tasks: Test of the lexical-processing hypothesis. European Journal of Cognitive Psychology, 17, 785-802. doi:10.1080/09541440440000212

Fernández-Rey, J., \& Merino, H. (2002). Sesgos de memoria implícita para información emocional en depresión subclínica (Implicit memory biases for emotional information in subclinical depression). Psicothema, 14, 795-801.

Fleischman, D. A. (2007). Repetition priming in aging and Alzheimer's disease: an integrative review and future directions. Cortex, 43, 889897. doi:10.1016/S0010-9452(08)70688-9

Geraci, L., \& Hamilton, M. (2009). Examining the response competition hypothesis of age effects in implicit memory. Aging, Neuropsychology, and Cognition, 16, 683-707. doi:10.1017/S1355617715001198

Gibson, J. M. (2012). Empirical investigations on priming of word-stem completion. In N. Hsu \& Z. Schütt (Eds.), Psychology of priming (pp. 1-48). New York: Nova Science Publishers, Inc.

Gibson, J. M., \& Watkins, M. J. (1988). A pool of 1.086 words with unique two-letter fragments. Behavior Research Methods, Instruments, \& Computers, 20, 390-397. doi:10.3758/BF03202683

Graf, P., \& Williams, D. (1987). Completion norms for 40 three-letter word stems. Behavior Research Methods, Instruments, \& Computers, 19, 422-445. doi:10.3758/BF03205611

Guasch, M., Boada, R., Ferré, P., \& Sánchez-Casas, R. (2013). NIM: A Webbased Swiss Army knife to select stimuli for psycholinguistic studies. Behavior Research Methods, 45, 765-771. doi:10.3758/s13428-0120296-8

Hartman, M., \& Pirnot, M. L. (1995). Indirect memory tests in Alzheimer's Disease. In P. Allen \& T. R. Bashore (Eds.), Age differences in word and language processing. Amsterdam: Elsevier.

Jacoby, L. L., Toth, J. P., \& Yonelinas, A. P. (1993). Separating conscious and unconscious influences of memory: Measuring recollection. Journal of Experimental Psychology: General, 122, 139-154. doi:10.1037/0096-3445.122.2.139

Keane, M. M., Gabrieli, J. D. E., Fennema, A. C., Growdon, J. H., \& Corkin, S. (1991). Evidence for a dissociation between perceptual and conceptual priming in Alzheimer's disease. Behavioral Neuroscience, 105, 326-342. doi:10.1037/0735-7044.105.2.326

Kellerman, B. A. (2002). Effects of emotionality on implicit and explicit memory for verbal stimuli. Dissertation Abstracts International: Section B: The Sciences and Engineering, 63, 2094.

Lang, P. J. (1980). Behavioral treatment and bio-behavioral assessment: Computer applications. In J. B. Sidowski, J. H. Johnson, \& T. A. Williams (Eds.), Technology in mental health and delivery systems (pp. 119-137). Norwood, NJ: Ablex.

MacLeod, C. M. (1989). Word context during initial exposure influences degree of priming in word fragment completion. Journal of Experimental Psychology: Learning, Memory, and Cognition, 15, 398-406. doi:10.1037/0278-7393.15.3.398

MacLeod, C. M., \& Kampe, K. E. (1996). Word frequency effects in recall, recognition, and word fragment completion tests. Journal of Experimental Psychology: Learning, Memory, and Cognition, 22, 132-142. doi:10.1037/0278-7393.22.1.132

Marques, V. S., Spataro, P., Cestari, V., Sciarretta, A., \& Rossi-Arnaud, C. (2016). Testing the identification/production hypothesis of implicit memory in schizophrenia: The role of response competition. Journal of the International Neuropsychological Society, 22, 314 321. doi:10.1017/S1355617715001198

Martin, S., Dressaire, D., Guerdoux, E., Trouillet, R., Brouillet, D., Brouillet, P., \& Maury, P. (2009). Norme francophone de complétion de trigrammes chez des participant âgés de 30 à 93 ans (French word-stem completion norms by participants from 30 to 93 
years old). European Review of Applied Psychology, 59, 25-36. doi:10.1016/j.erap.2008.09.001

Marvel, C. L., Schwartz, B. L., \& Isaacs, K. L. (2004). Word production deficits in schizophrenia. Brain and Language, 89, 182-191. doi:10.1016/S0093-934X(03)00366-3

Mathews, A., Mogg, K., May, J., \& Eysenck, M. (1989). Implicit and explicit memory bias in anxiety. Journal of Abnormal Psychology, 98, 236-240. doi:10.1037/0021-843X.98.3.236

Migo, E. M., Roper, A., Montaldi, D., \& Mayes, A. R. (2010). British English norms for the spontaneous completion of three-letter word stems. Behavior Research Methods, 42, 470-473. doi:10.3758/BRM.42.2.470

Millet, X., Le Goff, M., Bouisson, J., Dartigues, J. F., \& Amieva, H. (2010). Encoding processes influence word-stem completion priming in Alzheimer's disease: A meta-analysis. Journal of Clinical and Experimental Neuropsychology, 32, 494-504. doi:10.1080/13803390903224936

Mitchell, D. B., \& Bruss, P. J. (2003). Age differences in implicit memory: Conceptual, perceptual, or methodological? Psychology and Aging, 18, 807-822. doi:10.1037/0882-7974.18.4.807

Mueller, S. T., \& Thanasuan, K. (2014). Associations and manipulations in the mental lexicon: A model of word-stem completion. Journal of Mathematical Psychology, 59, 30-40. doi:10.1016/j.jmp.2014.01.001

Nebes, R. D. (1989). Semantic memory in alzheimer's disease. Psychological Bulletin, 106, 377-394. doi:10.1037/00332909.106.3.377

Nelson, D. L., \& McEvoy, C. L. (2000). What is this thing called frequency? Memory and Cognition, 28, 509-522. doi:10.3758/BF03201241

Nyberg, L., Winocur, G., \& Moscovitch, M. (1997). Correlation between frontal lobe functions and explicit and implicit stem completion in health elderly. Neuropsychology, 11, 70-76. doi:10.1037/08944105.11.1.70

Olofsson, U., \& Nyberg, L. (1992). Swedish norms for completion of word stems and unique word fragments. Scandinavian Journal of Psychology, 33, 108-116. doi:10.1111/j.1467-9450.1992.tb00890.x

Osgood, C., Suci, G., \& Tannenbaum, P. (1957). The measurement of meaning. Urbana, IL: University of Illinois.

Ostergaard, A. L. (1998). The effects on priming of word frequency, number of repetitions, and delay depend on the magnitude of priming. Memory and Cognition, 26, 40-60. doi:10.3758/BF03211369

Pomarol-Clotet, E., Oh, T. M. S. S., Laws, K. R., \& Mckenna, P. J. (2008). Semantic priming in schizophrenia: systematic review and meta-analysis. The British Journal of Psychiatry, 192, 92-97. doi:10.1192/bjp.bp.106.032102

Rajaram, S., \& Roediger, H. L., III. (1993). Direct comparison of four implicit memory tests. Journal of Experimental Psychology Learning, Memory, \& Cognition, 19, 765-776. doi:10.1037/02787393.19.4.765
Redondo, J., Fraga, I., Comesaña, M., \& Perea, M. (2005). Estudio normativo del valor afectivo de 478 palabras españolas (Normative Study of the emotional dimension of 478 Spanish words). Psicológica, 26, 317-326.

Roediger, H. L., III, \& Blaxton, T. A. (1987). Effects of varying modality, surface features, and retention interval on priming in word-fragment completion. Memory and Cognition, 15, 379-388. doi:10.3758/BF03197728

Roediger, H. L., III, Weldon, M. S., Stadler, M. L., \& Riegler, G. L. (1992). Direct comparison of two implicit memory tests: Word fragment and word stem completion. Journal of Experimental Psychology: Learning, Memory, and Cognition, 18, 1251-1269. doi:10.1037/0278-7393.18.6.1251

Royal Spanish Academy of Sciences. (1999). Diccionario Esencial de las Ciencias (Essential Dictionary of Sciences). Madrid: Espasa.

Royal Spanish Academy of Language. (2001). Diccionario de la Lengua Española (Dictionary of the Spanish Language) (22nd ed.). Madrid: Espasa.

Royal Spanish Academy of Language. (2011). Nueva gramática de la Lengua Española. Morfología (New grammar of the Spanish language. Morphology) (vol. 1). Madrid: Espasa.

Ryan, L., Ostergaard, A., Norton, L., \& Johnson, J. (2001). Search and selection processes in implicit and explicit word-stem completion performance in young, middle-aged, and older adults. Memory and Cognition, 29, 678-690. doi:10.3758/BF03200470

Soler, M. J., Dasí, C., \& Ruiz, J. C. (2009). Datos normativos de 269 fragmentos de palabras españolas a partir de la base de Dasí, Soler y Ruiz (2004) (Normative data of 269 fragments of Spanish words from the Dasi, Soler and Ruiz (2004) base). Psicológica, 30, $91-$ 117.

Soler, M. J., Dasí, C., \& Ruiz, J. C. (2015). Priming in word stem completion tasks: comparison with previous results in word fragment completion tasks. Frontiers in Psychology, 6, 1-8. doi:10.3389/fpsyg.2015.01172

Soler, M. J., Ruiz, J. C., Vargas, M., Dasí, C., \& Fuentes, I. (2011). Perceptual priming in schizophrenia evaluated by word fragment and word stem completion. Psychiatry Research, 190, 167-171. doi:10.1016/j.psychres.2011.08.008

Spataro, P., Cestari, V., \& Rossi-Arnaud, C. (2011). The relationship between divided attention and implicit memory: A meta-anaysis. Acta Psychologica, 136, 329-339. doi:10.1016/j.actpsy.2010.12.007

Spataro, P., Mulligan, N., \& Rossi-Arnaud, C. (2010). Effects of divided attention in the word fragment completion task with unique and multiple solutions. European Journal of Cognitive Psychology, 22, 18-45. doi:10.1080/09541440802685979

Srinivas, K., \& Roediger, H. L., III. (1990). Classifying implicit memory tests: Category association and anagram solution. Journal of Memory \& Language, 29, 389-412. doi:10.1016/0749-596X(90)90063-6CP 